\title{
ANALISIS ECOMOMICO DE OPCIONES PRODUCTIVAS PARA PLANTACIONES DE Eucalyptus nitens EN EL SUR DE CHILE
}

\author{
Juan Carlos Valencia Baier (1) y Jorge Armando Cabrera Pérramon (2)
}

\section{RESUMEN}

Invertir en establecer y manejar plantaciones de Eucalyptus nitens para producir rollizos podados con alta proporción de madera libre de nudos para chapa o aserrio, conjuntamente con rollizos aserrables con nudo firme y madera pulpable, cada dia cobra mayor interès en productores e inversionistas forestales, frente a la opción netamente pulpable; sin embargo, dado su relativo reciente desarrollo en Chile, son escasos los antecedentes sobre la rentabilidad de esta alternativa, situación que este trabajo busca mitigar mediante un análisis económico para la realidad productiva del sur del país.

Con dicha finalidad, se desarrolló un modelo de actualización de flujos de caja, con el cual se estimó y comparó la rentabilidad de invertir en plantar $E$. nitens considerando: a) un "Régimen Clear" o "Manejo de Alto Valor", con edad de rotación de 20 años, incluyendo tres podas y dos raleos comerciales; y b) la opción de producir sólo madera rolliza pulpable. considerando para ello un flujo de caja de dos rotaciones de monte alto sucesivas, de 10 años cada una: forestación y reforestación. En ambos casos se estimó el Valor Neto Presente (VNP); el Ingreso Anual Equivalente (IAE) y la Tasa Interna de Retorno (TIR), para dos condiciones de productividad de sitio: alta (IMA $45 \mathrm{~m}^{3} /$ ha-año; IS 33) y media (IMA $30 \mathrm{~m}^{3} /$ haaño; IS 26), representativas del rango de crecimiento observado en el sur de Chile.

Además, se analizó la rentabilidad de la opción pulpable para dos rotaciones de monte alto de E. globulus, también bajo dos condiciones de sitio: 20 y $30 \mathrm{~m}^{3} / \mathrm{ha}$-año, representativas de una productividad media a alta para la especie.

Los resultados indican que al $8 \%$ de tasa de descuento y en sitios de alta productividad, el VNP de la opción de alto valor de $E$. nitens se estima en 1.322 y 2.343 US $\$ /$ ha, según se considere o no el costo del terreno, generando 1.226 US $\$ /$ ha sobre la opción de invertir en dos rotaciones pulpables con la misma especie. En un sitio de productividad media, el VNP de la opción de alto valor se estimó en 355 y 1.101 US \$ / ha, según se considere o no el costo del terreno, generando 792 US \$ / ha sobre la opción pulpable. En términos relativos, la rentabilidad de la opción de alto valor fluctuó entre 9,0 a $16,2 \%$ real anual según productividad de sitio y, si se considera o no el costo del terreno, rentabilidad que para la opción pulpable con $E$. nitens se estimó en el rango de 6,1 a $15 \%$ real anual.

(1). Ingeniero Forestal, Investigador de Proyectos, INFOR Sede Los Lagos, Valdivia, jvalenci@infor cl

(2). Mg (E) Economia Agraria, Ingeniero Forestal, Director de Proyectos, INFOR Sede Los Lagos, Valdivia. jcabrera@infor.cl 
Se concluye que la opción de alto valor exhibe una alta rentabilidad, muy superior a la opción pulpable, que de consolidarse los mercados para la madera de $E$. nitens, puede significar importantes retornos para pequeños y medianos propietarios forestales.

El análisis a nivel de rodal de la opción pulpable con E. globulus, determinó altas rentabilidades en los sitios evaluados, concluyendo que no debieran primar razones económicas para cambiar el uso del suelo por $E$. nitens, donde $E$. globulus logre crecer con un IMA sobre $20 \mathrm{~m}^{3} / \mathrm{ha}$-año.

Palabras clave: Eucalyptus nitens; E. globulus; plantaciones; manejo; rentabilidad.

\section{SUMMARY}

Investing in establishing and managing plantations of Eucalyptus nitens for solid wood products to produce pruned logs with high proportion of clear wood for appearance veneer or sawn timber; sawlogs with knots for structural and industrial products and pulpwood, is an option that each day takes more attention in forest producers and investors, set against the pulpwood plantation option, nevertheless, product of their relative recent development, are scarce the antecedents about profitability, situation that this study mitigating with an analysis and comparison of the stand economy of both options, for the southern productive reality of Chile.

It is developed a cash flow model for financial analysis with which was calculated and compared the profitability of invest in planting E. nitens considering: a) "Clear Wood Regime" or "Higher Value Management", with a twenty year rotation, including three pruning and two commercial thinning; and b) the option of producing only pulpwood, considering for it a cash flow of two successive ten year rotation pulpwood each one. In both cases was calculated the Net Present Value (NPV); Annual Equivalent Return (AER) and Internal Rate of Return (IRR), for two site productivity: high (MAI of $45 \mathrm{~m}^{3} / \mathrm{ha} / \mathrm{yr} ; \mathrm{SI} 33$ ) and medium (MAI of $30 \mathrm{~m}^{3} / \mathrm{ha} / \mathrm{yr} ; \mathrm{SI} 26$ ), representative of the rank of growth observed for the species in the South of Chile.

Additionally, was evaluated the profitability of pulpwood option for two rotations of $E$. globulus, considering an MAl in volume of 20 and $30 \mathrm{~m}^{3} / \mathrm{ha} / \mathrm{yr}$, representative of a mean to high site productivity for this species.

For a discount rates of $8 \%$ and in a site with high productivity, the NPV of the Higher Value Management of $E$. nitens was calculated in 1.322 and 2.343 US $\$ /$ ha, according to be considered or not the cost of the land, generating an increment in the NPV of 1.226 US $\$ /$ ha set against the option of investing in two pulpwood rotations with the same species. In a site with medium productivity, the option of higher value generates a NPV of 355 and 1.101 US \$ I ha, considering or not the cost of the land, generating an increment in the NPV of 792 US \$ I ha set against the pulpwood plantation option, indicating the economic convenience of invest with objectives of solid wood products with higher value utilization. The IRR of the higher value management with $E$. nitens fluctuates among the 9,0 to $16,2 \%$ real annual 
according to site productivity and if is considered or not the cost of the land, profitability that in the pulpwood option with $E$. nitens was calculated in 6,1 and $15 \%$.

It is concluded that the option of higher value with $E$. nitens is an alternative with high profitability, much better than the pulpwood plantation option, therefore, if a market for $E$. nitens sawlogs and veneer is consolidated, the higher value plantation option can produce important benefits for small and medium owners of the country.

The high profitability of the pulpwood plantation option with E. globulus, permits to conclude that should not exist economic reasons for the land uses change with $\mathrm{E}$. nitens where E. globulus can grow on $20 \mathrm{~m}^{3} / \mathrm{ha} / \mathrm{yr}$.

Key words: Eucalyptus nitens; E. globulus; plantations; clear wood regime; profitability 


\section{INTRODUCCION}

La superficie mundial de plantaciones de eucalipto se estima en 19,4 millones de hectáreas (Baso 2004), orientadas principalmente a la producción de madera pulpable y combustible en rotaciones de 8 a 14 años; sin embargo, los últimos 5 a 10 años, un porcentaje creciente de esta superficie ha ido cambiando su orientación productiva, para ser establecida, manejada y cosechada, por numerosas empresas, entre ellas algunas multinacionales, para producir madera rolliza libre de nudos y defectos para aserrio y chapas, aprovechando la oportunidad de mercado que determinan la restricción de oferta de maderas duras tropicales y el crecimiento proyectado de la demanda por productos de madera sólida de alto valor, en especial de las principales economias como EEUU, Japón, China y la Unión Europea (Flynn, 2005). El progreso evidenciado los últimos 5 años en genética, silvicultura, cosecha, técnicas de aserrio y secado de madera de eucalipto, ha fortalecido el desarrollo de esta opción, superando con ello numerosas barreras tecnológicas y, de paso, algunas concepciones arraigadas sobre una supuesta mala reputación del género para dichos usos.

De esta manera, además del mercado pulpable y, eventualmente, de la madera reconstituida o compuesta ${ }^{1}$, Flynn (2005); Nolan et al. (2005); Montagu et al. (2003) y Shield, (2002), identifican tres sectores de mercado para rollizos de plantaciones de eucaliptos: a) pallets y embalajes; b) madera aserrada y chapas de uso estructural; y c) rollizos podados con alta proporción de madera libre de nudos y defectos para usos en los que predomina lo estético, como chapas decorativas y madera aserrada clear. El primer mercado utiliza rollizos de baja calidad, sector en que los eucaliptos ya incursionan con éxito a nivel mundial, incluso en Chile con E. nitens; el segundo mercado, para uso estructural, requiere de rollizos de alta calidad con pocos nudos y defectos para alcanzar las exigencias requeridas por norma. Sin embargo, algunos autores estiman que los eucaliptos serian poco competitivos frente a la madera de coniferas, entre ellas Pinus radiata, que dominan este mercado (Montagu et al. 2003; Cabrera, 2003), no obstante, si podrian tener oportunidades en aplicaciones en que la resistencia y tamaño sean importantes, o eventualmente como madera laminada para uso estructural (Gaunt et al. 2003, McKenzie et al. 2003; Montagu et al. 2003; Shield, 2002), de hecho en Tasmania, Australia, la empresa Forest Enterprise of Australia produce madera aserrada para uso estructural, denominada $E c o A s h \circledast$, con plantaciones de $E$. nitens ${ }^{2}$. El tercer sector, de alto valor (appearance grade) para muebleria, pisos, molduras y chapas decorativas, se perfila como el mercado con las mejores oportunidades para madera de plantaciones de eucalipto y al que se apunta con $E$. nitens, caracterizado por ser un mercado estable y de altos precios, tradicionalmente satisfecho por maderas duras de especies tropicales, oferta que progresivamente comienza a ser más escasa. Flynn (2005) concluye que es inevitable que progrese el uso de alto valor de las plantaciones de eucalipto y emerja como un importante componente del negocio mundial de maderas del siglo 21, en usos y aplicaciones de la madera sólida y en productos de ingenieria como el LVL, OSB y LSL (Laminated Strand Lumber).

De hecho, en varios paises operan empresas de diverso tamaño que manejan plantaciones y transforman madera de eucalipto para chapas y madera aserrada. En Brasil

Los tableros MDF (Medium Density Fibreboard) y OSB (Oriented Strand Board) han demostrado ser una opción industrial interesante para algunos eucaliptos, entre ellos $E$. nitens.

${ }^{2} \mathrm{http} / / \mathrm{www}$.forestenterprise.com/downloads/FEA_Flier_web.pdf 
destacan Aracruz, Klabin, CAF Santa Bárbara y Boise Cascade; en Argentina Forestadora Tapebicua y MASISA; en España el Grupo ENCE; en Uruguay Cofusa-Urufor, Euforest (ENCE) y Colonvade (joint venture Wayerhauser/UBB); todas utilizando Eucalyptus grandis; en Sudáfrica la empresa Mondi está renovando sus instalaciones para procesar madera de sus plantaciones y en Australia las empresas Gunns Ltd, Forestry Tasmania y Forest Enterprise of Australia cultivan e industrializan plantaciones de E. nitens.

Se suma la investigación y desarrollo sobre cultivo y procesamiento de plantaciones de eucalipto para madera sólida, ejecutada por numerosos institutos y centros tecnológicos tales como LATU en Uruguay, INTA en Argentina, EMBRAPA en Brasil, CSIR y la Universidad de Stellenbosch en Sudáfrica, CIRAD Fôret en Francia, CIS-Madera en España, CSIRO y CRC-SPF en Australia, el Forest Research Institute Ltd. en Nueva Zelanda e INFOR en Chile.

Sin embargo, se trata de un mercado reciente, que según Flynn (2005); Nollan et al. (2005) y Donnelly et al. (2003), representa una oferta mundial de rollizos aserrables de eucalipto de plantaciones del orden de los 3 millones de $\mathrm{m}^{3}$ al año $\mathrm{o}^{3}$, no obstante se proyecta supere los 10 millones de $\mathrm{m}^{3}$ anuales a partir del año $2015,10 \%$ del cual correspondería a rollizos podados de alto valor. Los principales oferentes son Brasil, Uruguay, Argentina, Sudáfrica y Australia, paises en que se ha realizado manejo intensivo de las plantaciones a través de podas y raleos y en los cuales la rentabilidad anual promedio de invertir en este tipo de objetivo productivo se ha estimado en el rango de 12 a 24\% (Cubbage et al. 2005; Nolan et al. 2005; Donnelly et al. 2003), la cual, según Flynn (2005) supera en muchos casos la rentabilidad real anual que registran los principales fondos de pensiones a nivel internacional.

En el caso particular de la especie E. nitens, en Australia Candy y Gerrand (1997) han estimado que la rentabilidad de invertir en la opción de alto valor registra una TIR del $12 \%$ real anual en los mejores sitios de crecimiento, incluyendo en este análisis el costo de uso del suelo. Operativamente, la empresa Gunns Plantation Ltd. (GPL) de Australia ofrece en sus proyectos de inversión (Woodlot Project) una TIR después de impuestos de 13,8\% para el esquema de producción de madera para chapas y pulpa con E. nitens, considerando una rotación de 20 años, frente al $10,9 \%$ que ofrece para la opción netamente pulpable, considerando una rotación de 13 años ${ }^{4}$.

Chile detectó esta oportunidad y aprovechando sus ventajas comparativas para el cultivo de eucaliptos, en especial con $E$. nitens, ha iniciado líneas de investigación aplicada, lideradas por el Instituto Forestal, INFOR, a través del proyecto FDI/CORFO "Desarrollo de Opciones Productivas de Mayor Valor para Plantaciones de Eucalyptus nitens en la IX y X Región", ejecutado entre los años 2002 y 2004, en asociación con empresas y productores forestales $y_{\text {; }}$ ahora con un nuevo proyecto FDI CORFO sobre investigación aplicada en silvicultura para producir rollizos de alto valor, estudio que se ejecutará ente los años 2005 al 2007.

\footnotetext{
${ }^{3}$ De un total de 6 miliones de $\mathrm{m}^{3}$, al considerar la oferta proveniente de bosques nativos australianos.

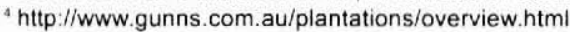


Avalan esta nueva opción productiva la experiencia reciente de algunas importantes empresas del pais, interesadas en diversificar el aprovechamiento industrial de $E$. nitens, incursionando en madera sólida, como lo demuestran las exportaciones del año 2004 y de enero - marzo de 2005 entre las que figuran los primeros envios de madera aserrada en tablones realizados por CMPC Maderas S.A., involucrando $1.863 \mathrm{~m}^{3}$ y un monto total de US $\$ 242$ mil FOB (INFOR, 2005a y 2005b). En el manejo de plantaciones, Forestal y Agrícola Monteáguila S.A. tiene proyectado el raleo y poda de 6 mil hectáreas de $E$. nitens de su patrimonio (Herranz, 2005), a lo cual se suma una superficie similar que un grupo de productores del sur del país tiene proyectado manejar para los próximos cinco años, en predios ubicados en las Regiones IX y X (Ludwig, 2005).

De esta forma, se abre para Chile una nueva opción de inversión y transformación industrial, representada por el manejo intensivo de plantaciones desde temprana edad, aprovechando el potencial de crecimiento y respuesta al manejo de esta especie y la oportunidad de acceder a un creciente mercado mundial en torno a los eucaliptos. Para los propietarios forestales representa una interesante opción de inversión, más aún en un escenario en que los precios de la madera pulpable de la especie limitan el desarrollo del negocio sólo a una determinada combinación de condiciones de sitio, proximidad a centros de consumo y escala de operaciones, entre otras (INFOR 2004a). De masificarse en Chile la opción de alto valor para $E$. nitens, significaria que las rotaciones originalmente planificadas sólo para producir madera de aptitud pulpable en turnos cortos, pueden orientarse a la producción conjunta de rollizos para chapas, aserrio y pulpa, con rotaciones de 15 a 20 años según sitio, silvicultura, tecnologías, productos y otras consideraciones relacionadas con la calidad de la madera, por lo cual se hace indispensable dimensionar su desempeño económico. En este contexto, el presente trabajo se plantea los siguientes objetivos:

\section{OBJETIVO GENERAL}

Contribuir con antecedentes e información sobre rentabilidad de invertir en establecer y manejar plantaciones de $E$. nitens para producir rollizos podados para chapa y aserrio como producto principal y, complementariamente, rollizos aserrables con nudo y pulpables, para las condiciones de crecimiento y costos del sur de Chile.

\section{OBJETIVOS ESPECIFICOS}

Calcular los indicadores de rentabilidad Valor Neto Presente (VNP), Ingreso Anual Equivalente (IAE) y Tasa Interna de Retorno (TIR), para la opción de alto valor con $E$. nitens y de su opción pulpable, bajo dos condiciones de productividad de sitio; con y sin considerar el costo de uso del suelo.

Calcular la rentabilidad de la opción pulpable con E. globulus y compararla con las opciones de alto valor y pulpable de $E$. nitens.

Determinar precios de equilibrio para la madera pulpable de $E$. nitens con los cuales se logra la indiferencia entre invertir en la opción pulpable y la de alto valor con esta especie, y entre la opción pulpable con $E$. nitens y $E$. globulus. 


\section{MATERIAL Y METODO}

\section{Modelo Económico}

Para construir los flujos de caja y estimar los indicadores de rentabilidad, se elaboró una planilla de cálculo en Microsoft Excel, considerando la alternativa de invertir en forestación con E. nitens para dos opciones productivas: a) Alto Valor, orientada a la producción de rollizos podados, aserrables con nudo firme y pulpable, con una rotación de 20 años, y b) Pulpable, considerando dos rotaciones, cada una de 10 años ${ }^{5}$. Adicionalmente, para efectos comparativos, el modelo se estructuró para permitir el análisis costo - beneficio de invertir en la opción pulpable con la especie $E$. globulus, considerando también un flujo de caja de dos rotaciones de monte alto de 10 años cada una ${ }^{6}$. Los indicadores Valor Neto Presente (VNP), Ingreso Anual Equivalente (IAE), ambos en dólares ${ }^{7}$ y la Tasa Interna de Retorno (TIR), fueron estimados empleando las siguientes formulas, obtenidas de Klemperer (1996) y Hubbard et al. (1998).

Valor Neto Presente:

$$
V N P=\sum_{y=0}^{\prime} \frac{I_{y}}{(1+i)^{y}}-\sum_{y=0}^{\prime} \frac{C_{y}}{(1+i)^{y}}
$$

Ingreso Anual Equivalente:

$$
I A E=\left\{\frac{V N P \times i(1+i)^{y}}{\left[(1+i)^{y}-1\right]}\right\}
$$

Tasa Interna de Retorno:

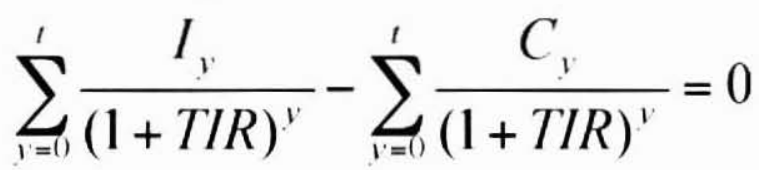

En que:

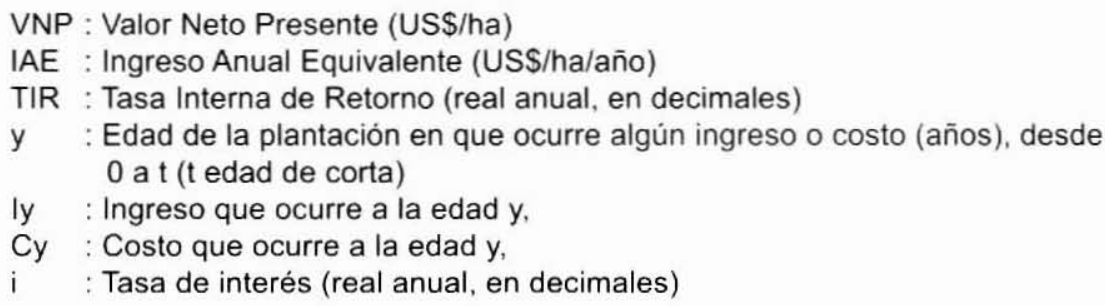

\footnotetext{
SSe asumen dos rotaciones de 10 años cada una, a objeto de que sean comparables con la opción de alto valor, sobre la base de un mismo periodo de inversión de 20 años.

${ }^{6}$ Se asume una segunda rotación de monte alto, considerando que en 10 años, la calidad genética de las plantas puede llegar a justificar esta opción frente al manejo de los retoños.

'Tipo de cambio observado 1 US\$ = \$581, promedio marzo-abril-mayo de 2005 (www.bcentral.cl)
} 
En todos los casos analizados se determinan los indicadores de rentabilidad para dos productividades de sitio: alta y media, que para $E$. nitens representa el rango de crecimiento observado en la región de Los Lagos. Los indicadores se estiman con y sin considerar el costo de uso del terreno (compraventa), pero no se incluye en el análisis el impacto de eventuales bonificaciones por forestación y manejo. Con respecto a la tasa de descuento, tanto el VNP como el IAE se estimaron considerando un $8 \%$ real anual, magnitud razonable como costo de oportunidad del capital para proyectos forestales que involucran largos periodos de maduración.

\section{Opciones Evaluadas}

Para la opción de alto valor se consideró un "esquema tipo" con rotación de 20 años, derivado de: información generada en el Proyecto FDI CORFO INFOR sobre Industria y Mercado, ejecutado el 2002-04 (INFOR, 2004a); información de esquemas australianos (Gerrand et al. 1997); antecedentes recopilados en una gira nacional e internacional, realizadas por el Grupo Nitens de Chile (INFOR 2004b, 2003a); la práctica de varios productores de la región de Los Lagos vinculados a proyectos de INFOR y antecedentes de Forestal Mininco S.A. (Dunn, 2003). En la opción pulpable, tanto para $E$. nitens como $E$. globulus, se asume la práctica actual, con rotaciones de 10 años. El Cuadro $N^{\circ} 1$ detalla las actividades para ambas opciones, consideradas en el modelo de evaluación. En la opción de alto valor, se aprecia que la oportunidad de los raleos comerciales difiere según productividad del sitio evaluada (alta y media). En el sitio de alta productividad, el primer y segundo raleo comercial se asumen a la edad de 5 y 10 años, respectivamente; y en el sitio de productividad media, a los 6 y 11 años, respectivamente. 


\section{Cuadro $\mathrm{N}^{\circ} 1$ \\ DESCRIPCION DE ACTIVIDADES SEGUN OPCIONES PRODUCTIVAS EVALUADAS}

\begin{tabular}{|c|c|c|}
\hline Ano & Pulpable* & Alto Valor \\
\hline 0 & $\begin{array}{l}\text { Habilitación, preparación del suelo, cercado } \\
\text { Control de malezas pre-plantación } \\
\text { Plantacion de } 1.429 \text { plantasina } \\
\text { Fertilización (formulación general completa) } \\
\text { Control quimico de malezas post plantación }\end{array}$ & $\begin{array}{l}\text { Habilitacion. preparación del suelo, cercado } \\
\text { Control de malezas pre-plantación } \\
\text { Plantación de } 1.429 \text { plantas/ha } \\
\text { Fertilización (formulación general completa) } \\
\text { Control químico de malezas post plantación }\end{array}$ \\
\hline $1-2$ & Control quimico de malezas mantenimiento & Control químico de malezas mantenimiento \\
\hline 3 & - & $\begin{array}{l}\text { Poda: mejores } 700 \text { arthiha hasta una altura libre de } \\
\text { ramas de } 2.5 \mathrm{~m}\end{array}$ \\
\hline 4 & - & Primer levante poda: 500 arb/ha hasta $4,5 \mathrm{~m}$ \\
\hline 5 & $\cdot$ & $\begin{array}{l}\text { Segundo levante poda: } 300 \text { artha a } 7.5 \mathrm{~m} \\
\text { Primer raleo comercial en sitios de alta productividad. se } \\
\text { eliminan los árboles no podados ( } 729 \text { arb/ha) }\end{array}$ \\
\hline 6 & $\cdot$ & $\begin{array}{l}\text { Primer raleo comercial en sitios de productividad media: } \\
\text { se eliminan los árboles no podados (729 artha) }\end{array}$ \\
\hline 10 & $\begin{array}{l}\text { Cosecha primera rotación } \\
\text { Habilitación y preparación del suelo } \\
\text { Relorestación con } 1.429 \text { plantas/ma } \\
\text { Fertilización (formulación general completa) }\end{array}$ & $\begin{array}{l}\text { Segundo raleo comercial en sitios de alta productividad: } \\
\text { quedan } 300 \text { artha podados (se eliminan } 400 \text { art/ha) }\end{array}$ \\
\hline 11 & Control quimico de malezas mantenimiento & $\begin{array}{l}\text { Segundo raleo comercial en sitos de productrvidad } \\
\text { media: quedan } 300 \text { artha podados (se eliminan } 400 \\
\text { arb/ha) }\end{array}$ \\
\hline 12 & Control quimico de malezas mantenimiento & 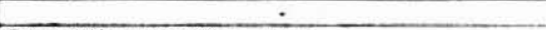 \\
\hline 1 a 20 & Supervision, protección, seguros & Supervisión, protección, seguros \\
\hline 20 & Cosecha segunda rotación & Cosecha final \\
\hline
\end{tabular}

- Tanio para $E$. nitens como $E$. globulus, se emplea esta estructura productiva

En ambas opciones productivas se asume una misma densidad y tecnologia de establecimiento, no obstante la opción de alto valor tiene por objetivo principal producir rollizos con madera libre de nudos, para lo cual considera la ejecución de 3 podas con periodicidad anual, logrando una altura final libre de ramas de $7,5 \mathrm{~m}$ y 2 raleos comerciales, considerando una edad de cosecha 20 años, asumiendo que en las productividades de sitio analizadas, los árboles logran un DAP $\geq 40 \mathrm{~cm}$, que satisface los requerimientos industriales para producir dos rollizos libres de nudos por árbol, uno clear o multipropósito de $4 \mathrm{~m}$ y un rollizo debobinable de $2,8 \mathrm{~m}$ de largo, con un diámetro menor mínimo de $28 \mathrm{~cm}$ (JAS). Para rollizos aserrables nudosos, se asume un producto industrial de largo $3,35 \mathrm{~m}$ y $18 \mathrm{~cm}$ JAS de diámetro menor minimo ${ }^{8}$.

\footnotetext{
${ }^{B}$ En ausencia de especificaciones para Eucalipto se empleó una norma para $P$. radiata (Mininco 2003)
} 


\section{Productividad de Sitio y Rendimientos}

Según datos observados por productores y empresas de la región de Los Lagos, se estima que el rango probable de incremento medio anual en volumen (IMA) para plantaciones de $E$. nitens sin manejo, evaluado a los 10 años de edad, fluctúa entre los 30 y $45 \mathrm{~m}^{3} / \mathrm{ha}$ año $0^{9}$, valores consistentes con las proyecciones de los modelos de crecimiento disponibles (INFOR, 2000; Zapata, 2001). En base a las funciones del modelo de crecimiento Euca3.2 por especie, desarrollado por INFOR y considerando una densidad de establecimiento de $1.429 \mathrm{arb} / \mathrm{ha}$, dichas productividades son similares a las registradas en indices de sitio (IS) de 26 y 33 metros. Para la opción de alto valor, en Chile no existen herramientas que simulen el crecimiento o información publicada de rendimientos observados de rodales con poda y raleo, aspecto que INFOR abordará con su nuevo proyecto de silvicultura para la especie. Para superar esta limitante, en este trabajo se asumen volúmenes comerciales para ambos raleos según antecedentes productivos de experiencias de manejo en la región de Los Lagos, validadas con proyecciones del simulador Euca3.2 según volumen medio por árbol a la edad del raleo y número de árboles extraídos. Para determinar el volumen de cosecha final de la opción de alto valor, se asumió que el volumen total acumulado, incluido raleos, fuese el $90 \%$ del volumen acumulado de dos rotaciones pulpables, considerando el IMA de la primera rotación. Para este volumen de cosecha final, en ambos sitios, se asumió que el $40 \%$ es aptitud pulpable; $30 \%$ aserrable nudoso y $30 \%$ rollizos podados (Cuadro $\mathrm{N}^{\circ} 2$ ).

\section{Cuadro $\mathrm{N}^{\circ} 2$}

\section{RENDIMIENTOS EVALUADOS SEGUN SITIO Y OPCION PRODUCTIVA PARA E. nitens (m ${ }^{3 / h a)}$}

\begin{tabular}{|l|c|c|c|c|c|c|}
\hline \multicolumn{1}{|c|}{ SITIO } & \multicolumn{2}{|c|}{ MEDIA PRODUCTIVIDAD (IS 26) } & \multicolumn{2}{c|}{ AL TA PRODUCTIVIDAD (IS 33) } \\
\hline OPCIÓN & Alto Vaior & \multicolumn{2}{|c|}{ Pulpable } & Alto Valor & \multicolumn{2}{c|}{ Puipabie } \\
\hline Variables Fisicas & & $2^{2}$ rotación & $2^{2}$ rotación & & $1^{2}$ rotación & $2^{\circ}$ rotación \\
\hline Volumen raleo 1 & 50 & 0 & 0 & 60 & 0 & 0 \\
\hline Volumen raleo 2 & 100 & 0 & 0 & 110 & 0 & 0 \\
\hline IMA edad de corta & 19.5 & 30 & 33 & 32 & 45 & 49.5 \\
\hline Volumen cosecha final & 390 & 300 & 330 & 640 & 450 & 495 \\
\hline Pulpable & 156 & 300 & 330 & 256 & 450 & 495 \\
\hline Aserrabie con nudo & 117 & 0 & 0 & 192 & 0 & 0 \\
\hline Podado & 117 & 0 & 0 & 192 & 0 & 0 \\
\hline
\end{tabular}

En ambos sitios se asume que la segunda rotación pulpable logra un 10\% mas de volumen comercial que la primera, producto de avances genéticos, tecnologicos y silvicolas.

Para la opción pulpable con E. globulus, se asumió un IMA de 20 y $30 \mathrm{~m}^{3} / \mathrm{ha} /$ año, según productividad de sitio media y alta, respectivamente ${ }^{10}$, crecimiento que en base a las proyecciones del simulador Euca3.2 equivalen a un IS de 22 y 28 , respectivamente, para una densidad inicial de $1.429 \mathrm{arb} / \mathrm{ha}$. Al igual que en $E$. nitens, la segunda rotación pulpable se asume un IMA un $10 \%$ superior a la primera rotación. Estos antecedentes de crecimiento son consistentes con información proporcionada por Prado y Barros (1989), para la Región Oceánica de Los Lagos, delimitada por los paralelos $39^{\circ}$ y $42^{\circ} \mathrm{S}$, quienes mencionan proyecciones de crecimiento para $E$. globulus spp globulus en el rango de 10 a $29 \mathrm{~m}^{3} / \mathrm{ha}$ año. De acuerdo a Geldres y Schlatter (2004), el potencial de E. globulus, de $32,1 \mathrm{~m}^{3} / \mathrm{ha} /$

\footnotetext{
${ }^{9}$ Incluso en determinados sitios y parcelas se han observado /IMA superiores a los $45 \mathrm{~m}^{3} / \mathrm{ha} / \mathrm{año}$.

${ }^{10}$ En sitios desfavorables y técnicas poco adecuadas en el establecimiento de E. globulus, Geldres y Schlatter (2004) calcularon un IMA de 10 a $12 \mathrm{~m}^{3} / \mathrm{ha}$ - año en plantaciones de la provincia de Osorno.
} 
año, medido en una plantación establecida con técnicas adecuadas y ubicada en un sitio favorable, corresponde al crecimiento máximo proyectado para esta zona.

\section{Costos de Establecimiento y Manejo}

Según información de productores de la región de Los Lagos y los antecedentes de costos de forestación publicados por CONAF (2004) para la temporada 2005, el Cuadro $N^{\circ} 3$ entrega la magnitud de costos medios de establecimiento y podas empleados en este trabajo.

\section{Cuadro $\mathrm{N}^{\circ} 3$ \\ SUPUESTOS SOBRE COSTOS DE ESTABLECIMIENTO, MANTENIMIENTO Y PODA DE PLANTACIONES DE EUCALIPTO SEGUN OPCION PRODUCTIVA}

(US\$/ha)

\begin{tabular}{|c|c|c|c|c|c|c|}
\hline \multirow[t]{3}{*}{ Actividad } & \multirow{2}{*}{\multicolumn{2}{|c|}{ Opción de Alto Valor }} & \multicolumn{4}{|c|}{ Opción Pulpable } \\
\hline & & & \multicolumn{2}{|c|}{ Forestación } & \multicolumn{2}{|c|}{ Reforestación } \\
\hline & Año & (USS/ha) & Aก๊o & (US\$/ha) & Aก̃o & (USS/ha) \\
\hline $\begin{array}{l}\text { Habilitación. cercado y preparación } \\
\text { terreno }\end{array}$ & 0 & 260 & 0 & 260 & 10 & $150^{\circ}$ \\
\hline Control malezas pre-plantación & 0 & 80 & 0 & 80 & 10 & 0 \\
\hline Plantas y plantación & 0 & 160 & 0 & 160 & 10 & 160 \\
\hline Control malezas post-plantación & 0 & 75 & 0 & 75 & 10 & 75 \\
\hline Fertización & 0 & 110 & 0 & 110 & 10 & 80 \\
\hline Control malezas mantenimiento & $1-2$ & 80 & 1.2 & 80 & 11.12 & 80 \\
\hline $\begin{array}{l}\text { Costos anvales administración. } \\
\text { proteccion y seguros }\end{array}$ & 1 al 20 & 20 & 1 al 10 & 20 & 11 al 20 & 20 \\
\hline Primera poda & 3 & 75 & . & $\cdot$ & . & . \\
\hline Segunda poda & 4 & 80 & - & - & - & $\cdot$ \\
\hline Tercera poda & 5 & 85 & $\cdot$ & - & $\cdot$ & - \\
\hline
\end{tabular}

- No considera cercado pero incluye fajeo de residuos de cosecha en curvas de nivel

Fuente: elaboración propia segùn información proporcionada por Menzel (2005). Leiva (2004) y Ludwig (2004).

\section{Costo de Adquisición y Precio de Venta del Suelo}

Para incluir el costo de uso del suelo se empleó precios de la tierra diferenciados según productividad de sitio: 950 y $1.300 \mathrm{US} \$ /$ ha, para sitios de productividad media y alta, respectivamente, que son promedios representativos del valor que, a la fecha, alcanzan los suelos de aptitud forestal en el sur del pais (Ludwig, 2005). En ambos casos se asume el mismo precio real de compra (al año 0 ) y venta (al año 20 ), con lo cual se considera sólo los intereses que se dejan de percibir por este concepto.

\section{Costos de Cosecha y Transporte}

Según antecedentes proporcionados por productores del Grupo Nitens (Bregar 2005: Ludwig, 2005; Menzel, 2005) y de cálculos realizados por Provoste (2004) para operaciones de cosecha de $E$. nitens, se empleó un costo de $15,7 \mathrm{US} \$ / \mathrm{m}^{3} \mathrm{ssc}$ como valor promedio unitario, el cual considera cosecha y carguio por $7,2 \mathrm{US} \$ / \mathrm{m}^{3}$ y $8,5 \mathrm{US} \$ / \mathrm{m}^{3}$ para transporte. asumiendo un flete corto de $5 \mathrm{Km}$ y un flete de $80 \mathrm{Km}$ desde el bosque al centro de consumo. Si bien en una misma plantación pueden existir diferencias entre costos unitarios para la extracción de madera de raleo y cosecha final y según la dimensión de los productos (chapa, 
aserrio y pulpa), este trabajo las omite para simplificar la estructura del modelo económico y el análisis".

\section{Precio de Productos}

El análisis económico de ambas opciones productivas, considera la venta de rollizos puestos en planta, asignando para ello los siguientes precios por tipo de producto: $\$ 21.000$ por metro ruma de madera rolliza pulpable de $E$. nitens, lo que equivale a $23,4 \mathrm{US} \$ / \mathrm{m}^{3} \mathrm{ssc}^{12}$, y que es consistente con las estadisticas de precio publicadas por INFOR (2003b) y con el precio del mercado interno que están recibiendo algunos productores de la región de Los Lagos.

Para los rollizos aserrables con nudo y podados de $E$. nitens, dado que no existe un mercado interno establecido, como supuesto se asume los precios puesto planta para la especie pino radiata: 35 y $55 \mathrm{US} \$ / \mathrm{m}^{3}$, respectivamente. Al respecto, es importante mencionar que el precio que recibirá el productor por sus rollizos de alta calidad de $E$. nitens, dependerá de una serie de factores, por lo que no es factible predecirlo; sin embargo, al emplear la magnitud y estructura de precios del mercado interno del pino radiata como referencia, debiese interpretarse como un escenario prudente, pero conservador para una madera de latifoliada que aspira a acceder a usos de alto valor.

Para la madera pulpable de $E$. globulus se asumió un precio puesto planta de $\$ 31.500$ por metro ruma, lo que equivale a $35 \mathrm{US} \$ / \mathrm{m}^{3} \mathrm{ssc}$ puesto planta ${ }^{13}$.

En base a los supuestos de costos de cosecha - transporte y los precios puesto planta, en el Cuadro $\mathrm{N}^{\circ} 4$ se consolida los precios de madera en pie utilizados en el análisis de rentabilidad de las opciones descritas.

\section{Cuadro $\mathrm{N}^{\circ} 4$}

PRECIO EN PIE POR TIPO DE PRODUCTO Y OPCION DE MANEJO (US\$/m $\mathbf{s s c}$ )

\begin{tabular}{|l|c|c|c|}
\hline Rollizo & $\begin{array}{c}\text { E. nitens } \\
\text { Opción Alto valor }\end{array}$ & $\begin{array}{c}\text { E. nitens } \\
\text { Opción Pulpable }\end{array}$ & $\begin{array}{c}\text { E. globulus } \\
\text { Opción Pulpable }\end{array}$ \\
\hline Pulpable & 7.7 & 7.7 & 19,3 \\
\hline Aserrable nudoso & 19,3 & - & - \\
\hline Podado & 39,3 & - & - \\
\hline
\end{tabular}

\footnotetext{
"Para la opción de alto valor se han omitido posibles costos de pre y post cosecha por eventuales tratamientos orientados a mitigar el efecto de las tensiones de crecimiento de la madera tales como anillamiento en pie y uso de ceras emulsionantes para el sellado de las trozas, puesto que a la fecha no se dispone de antecedentes para Chile que efectivamente demuestren su conveniencia.

${ }^{12}$ Considera un factor de conversión de $1.55 \mathrm{~m}^{3}$ por cada metro ruma

${ }^{13}$ Zapata (2001) evaluando la rentabilidad de diferentes opciones de establecimiento, empleó en su análisis precios puesto planta para el metro ruma pulpable de $\$ 20$ mil para $E$. nitens y de $\$ 30$ mil para $E$. globulus.
} 


\section{RESULTADOS}

\section{Sitio de Alta Productividad}

En el Cuadro $N^{\circ} 5$ se proporciona los resultados de indicadores de rentabilidad estimados para una rotación de 20 años de $E$. nitens bajo esquema de alto valor; para dos rotaciones de 10 años con manejo pulpable de $E$. nitens y para dos rotaciones con manejo pulpable de $E$. globulus. En el caso de $E$. nitens, los indicadores estimados corresponden a IS 33 y en E. globulus a IS 28 .

\section{Cuadro $N^{\circ} 5$}

INDICADORES DE RENTABILIDAD PARA PLANTACIONES DE E. nitens y E. globulus SEGUN OPCION PRODUCTIVA, CON Y SIN CONSIDERAR EL COSTO DEL TERRENO.

\begin{tabular}{|c|c|c|c|c|c|c|}
\hline \multirow{2}{*}{ OPCION } & \multicolumn{3}{|c|}{ Considerando costo terreno } & \multicolumn{3}{c|}{ Sin considerar costo terreno } \\
\cline { 2 - 7 } & $\begin{array}{c}\text { VNP }(8 \%) \\
\text { (USs/ha) }\end{array}$ & $\begin{array}{c}\text { IAE (8\%) } \\
\text { (USS/ha/año) }\end{array}$ & $\begin{array}{c}\text { TIR } \\
(\%)\end{array}$ & $\begin{array}{c}\text { VNP (8\%) } \\
\text { (USS/ha) }\end{array}$ & $\begin{array}{c}\text { IAE }(8 \%) \\
\text { (USS/ha/aho) }\end{array}$ & $\begin{array}{c}\text { TIR } \\
\text { (\%) }\end{array}$ \\
\hline E. nitens Alto Valor & $\mathbf{1 . 3 2 2}$ & $\mathbf{1 3 5}$ & $\mathbf{1 0 , 8}$ & $\mathbf{2 . 3 4 3}$ & $\mathbf{2 3 9}$ & $\mathbf{1 6 , 2}$ \\
\hline E nitens Pulpable & $\mathbf{9 6}$ & 10 & $\mathbf{8 , 3}$ & $\mathbf{1 . 1 1 7}$ & $\mathbf{1 1 4}$ & $\mathbf{1 5 , 0}$ \\
\hline E. globulus Pulpable & $\mathbf{1 . 7 2 2}$ & $\mathbf{1 7 5}$ & $\mathbf{1 2 . 7}$ & $\mathbf{2 . 7 4 3}$ & $\mathbf{2 7 9}$ & $\mathbf{2 1 , 2}$ \\
\hline
\end{tabular}

\section{Sitio de Productividad Media}

El Cuadro $\mathrm{N}^{\circ} 6$ entrega resultados de indicadores de rentabilidad estimados para una rotación de 20 años de $E$. nitens bajo esquema de alto valor; para dos rotaciones de 10 años con manejo pulpable de $E$. nitens y para dos rotaciones con manejo pulpable de $E$. globulus. En el caso de $E$. nitens, los indicadores estimados corresponden a IS 26 y en $E$. globulus a IS 22 .

\section{Cuadro $\mathrm{N}^{\circ} 6$}

INDICADORES DE RENTABILIDAD PARA PLANTACIONES DE E. nitens y E. globulus SEGUN OPCION PRODUCTIVA, CON Y SIN CONSIDERAR EL COSTO DEL TERRENO

\begin{tabular}{|c|c|c|c|c|c|c|}
\hline \multirow{2}{*}{ OPCION } & \multicolumn{3}{|c|}{ Considerando costo terreno } & \multicolumn{3}{|c|}{ Sin considerar costo terreno } \\
\cline { 2 - 7 } & $\begin{array}{c}\text { VNP (8\%) } \\
\text { (USS/ha) }\end{array}$ & $\begin{array}{c}\text { IAE (B\%) } \\
\text { (USS/ha/año) }\end{array}$ & $\begin{array}{c}\text { TIR } \\
(\%)\end{array}$ & $\begin{array}{c}\text { VNP (8\%) } \\
\text { (USS/ha) }\end{array}$ & $\begin{array}{c}\text { IAE }(8 \%) \\
(\text { USS/ha/ano) }\end{array}$ & $\begin{array}{c}\text { TIR } \\
(\%)\end{array}$ \\
\hline E. nitens Alto Vaior & 355 & 36 & 9,0 & 1.101 & 112 & 12,8 \\
\hline E. nitens Pulpable & -437 & -44 & 6,1 & 310 & 32 & 10,4 \\
\hline E. globulus Pulpable & 647 & 66 & 10,4 & 1.393 & 142 & 16,3 \\
\hline
\end{tabular}

\section{Precio de la Madera Pulpable E. nitens para el Equilibrio}

Para una misma condición de sitio (alta o media) y con los supuestos utilizados, el Cuadro $\mathrm{N}^{0} 7$ proporciona los resultados del precio equilibrio para la madera pulpable de $E$. nitens puesto planta y en pie, con el cual se iguala el VNP (a un $8 \%$ real anual) de la opción pulpable frente a la opción de alto valor con esta especie, y el precio con que se logra la indiferencia entre invertir en manejo pulpable con E. nitens o con E. globulus (ceteris paribus), en este último caso en la eventualidad que ambos proyectos fueran mutuamente excluyentes. 


\section{Cuadro $\mathrm{N}^{\circ} 7$}

PRECIOS PARA LA INDIFERENCIA DE INVERTIR ENTRE OPCION PULPABLE CON E.nitens FRENTE A OPCION DE ALTO VALOR Y OPCION PULPABLE CON E.globulus.

\begin{tabular}{|c|c|c|}
\hline \multirow{2}{*}{$\begin{array}{c}\text { Precio Pulpable } \boldsymbol{E} \text {. nitens } \\
\text { (USS/m3 scc) }\end{array}$} & \multicolumn{2}{|c|}{ Para lgualar la Opción Productiva } \\
\cline { 2 - 3 } & E. nitens Alto Valor & E. globulus Pulpable \\
\hline Puesto Planta & 30,7 & 28,56 \\
\hline En Pie & 15 & 12,86 \\
\hline
\end{tabular}

\section{DISCUSION}

La opción de establecer y manejar plantaciones de $E$. nitens con el objetivo primario de producir rollizos podados para chapas y/o aserrio y de manera complementaria madera pulpable, considerando rotaciones de 20 años, es una opción rentable, incluso en sitios de productividad media, siendo una alternativa superior a la opción netamente pulpable. En sitios de alta productividad la TIR de la opción de alto valor fue de 10,8 y $16,2 \%$ real anual, con y sin considerar el costo de uso del suelo durante la rotación, respectivamente. EI VNP. para una tasa de descuento del $8 \%$, se estimó en 1.322 y 2.343 US\$/ha, según se considere o no el costo del suelo, determinando un ingreso anual equivalente de 135 y 239 US\$/ha, respectivamente.

En contraste la opción de invertir en dos rotaciones pulpables de 10 años cada una con la especie $E$. nitens en sitios de alta productividad, determina rentabilidades inferiores a la opción de alto valor, estimándose una TIR de 8,3 y $15 \%$ real anual, según se considere 0 no el costo del suelo, respectivamente. En términos absolutos, considerando una tasa de descuento del $8 \%$, la opción pulpable determina un VNP de 96 y 1.117 US\$/ha, con y sin considerar el costo del terreno. Se aprecia que en sitios de alta productividad, la diferencia en magnitud del valor actual de decidir destinar la plantación de $E$. nitens a un objetivo de mayor valor frente a la alternativa de dos rotaciones pulpables, significaria para el productor incrementar su riqueza actual en más de US\$1.220 por cada hectárea plantada.

En sitios de productividad media, la opción de alto valor con $E$. nitens determina una TIR de 9,0 y $12,8 \%$ según se considere o no el costo de uso del terreno. En estos sitios, la magnitud del VNP a una tasa de descuento del $8 \%$ real anual, se estima en 355 y 1.101 US\$/ha, con y sin considerar el costo del suelo, lo que es equivalente a una anualidad de 36 y 112 US\$/ha durante 20 años, respectivamente.

Invertir en dos rotaciones pulpables con E. nitens en sitios de productividad media en tanto, determina rentabilidades inferiores a la opción de alto valor, estimándose una TIR de 6,1 y $10,4 \%$ real anual, con y sin considerar el costo del suelo respectivamente. En términos absolutos, considerando una tasa de descuento del $8 \%$, la opción pulpable determina un VNP de -437 y 310 US\$/ha, con y sin considerar el costo del terreno. Esto significa que en sitios de productividad media, la diferencia en magnitud del valor actual de decidir destinar la plantación de $E$. nitens a un objetivo de mayor valor frente a la opción de dos rotaciones pulpables, significaría para el productor incrementar su riqueza actual en más de US\$790 por cada hectárea. 
Al comparar $E$. nitens con E. globulus, se aprecia que dos rotaciones pulpables de 10 años cada una con la especie $E$. globulus, creciendo a un IMA de $30 \mathrm{~m}^{3} / \mathrm{ha} / \mathrm{año}$, representan una inversión con mayor retorno frente a plantar $E$. nitens bajo un esquema de alto valor, y ambas muy superiores al manejo netamente pulpable con $E$. nitens.

No obstante, se debe tener presente que a diferencia de $E$. nitens, la especie $E$. globulus no prospera con éxito en terrenos con alta frecuencia e intensidad de heladas, situación en la cual no representaria una inversión alternativa. La comparación es correcta en sitios donde $E$. globulus y $E$. nitens se desarrollan bien, situación en que se concluye no debieran primar razones económicas para cambiar el uso de $E$. globulus a $E$. nitens, salvo que los precios futuros de los rollizos podados de $E$. nitens sean muy superiores a los precios empleados en este trabajo.

Las rentabilidades determinadas son consistentes con las referencias proporcionadas por Cubbage et al. (2005); INFOR (2004a) y Donnelly et al. (2003) para plantaciones de Eucalyptus sp en Sudamérica y Chile, y con los resultados de Candy y Gerrand (1997) para E. nitens en Australia. En las mejores condiciones de sitio y crecimiento, y aún con los precios conservadores utilizados para los rollizos podados, las rentabilidades estimadas para la opción de alto valor superan la rentabilidad anual promedio que han registrado los fondos de pensiones en Chile y de sus rentabilidades esperadas en el largo plazo. A modo de ejemplo, en el periodo 1981 - enero 2005, el promedio de rentabilidad real anual del fondo $C$ fue de $10,25 \%$ (SAFP, 2005), frente al $16 \%$ anual que renta el manejo de alto valor con E. nitens en sitios de alta productividad.

En sitios de alta productividad y sin considerar el costo de uso del terreno, la opción pulpable con $E$. nitens exhibe una alta rentabilidad, sin embargo al considerar el costo del suelo o cuando se establece en terrenos de mediana productividad, es una opción que se encuentra en el límite de la eficiencia económica para este tipo de inversiones, básicamente porque el precio actual de mercado determina un bajo precio de la madera en pie, muy sensible a los costos de flete, dejando un margen que aun con el notable crecimiento de la especie, no es suficiente en algunos casos para cubrir los costos de formación y dejar un beneficio neto atractivo para el inversionista. Por ejemplo, en sitios de alta productividad, la opción pulpable de $E$. nitens genera $450 \mathrm{~m}^{3} /$ ha a los 10 años, volumen 1,5 veces superior al registrado en plantaciones pulpables de $E$. globulus en sitios de alta productividad; no obstante, la diferencia en la rentabilidad, radica en que el precio en pie de $E$. globulus es 2,5 veces superior al $E$. nitens $\left(19,3 \mathrm{v} / \mathrm{s} 7,7 \mathrm{US} \$ / \mathrm{m}^{3}\right)$. Por ello, la opción pulpable con $E$. nitens será conveniente sólo en terrenos productivos ubicados cerca de los centros de consumo, evitando de esta manera altos costos de transporte que reducen el precio en pie.

En este contexto, y para una misma condición de sitio, se estimó que el precio puesto planta de la madera pulpable de $E$. nitens que iguala la rentabilidad de la opción pulpable de $E$. nitens con $E$. globulus, es de $28,56 \mathrm{US} \$ / \mathrm{m}^{3}$, lo que determina un precio en pie de 12,86 US $\$ / \mathrm{m}^{3}$, frente a los 19,3 de $E$. globulus. Es decir, que el precio en pie de $E$. globulus puede ser 1,5 veces mayor y aun asi se obtendria igual rentabilidad, ya que se mitiga por el mayor volumen de $E$. nitens, sin embargo hoy en dia este diferencial es muy superior. Similar análisis permitió determinar que con un precio de la madera pulpable de $E$. nitens de $30,7 \mathrm{US} \$ / \mathrm{m}^{3}$ puesto planta ( $\$ 27.600$ por MR ssc), el productor seria indiferente 
financieramente entre manejar o no las plantaciones de $E$. nitens, esto considerando que el mayor precio también beneficia a la fracción de volumen pulpable de la opción de alto valor.

Finalmente es conveniente recalcar que los supuestos empleados para la opción de alto valor con $E$. nitens, representan un escenario conservador, motivo por el cual los resultados expuestos podrian estar subestimando la rentabilidad de esta alternativa.

\section{CONCLUSIONES}

El manejo de alto valor de plantaciones de E. nitens, orientado a producir rollizos para aserrio, chapas y pulpa, es una opción productiva con alta rentabilidad, que frente a la opción pulpable permite un incremento en el VNP estimado en 1.220 a 790 US\$/ha, en sitios de productividad alta y media, respectivamente.

En sitios de alta productividad (IS 33), la opción de alto valor con E. nitens registra una TIR de 10,8 y $16,2 \%$, con y sin considerar el costo del terreno; un VNP ( $8 \%$ ) de 1.322 y 2.343 US\$/ha, con y sin incluir en el análisis el costo del suelo, respectivamente, lo que equivale a rentas anuales de 135 y 239 US $\$ /$ ha.

En sitios de productividad media (IS 26), la opción de alto valor con $E$. nitens registra una TIR de 9,0 y $12,8 \%$, con y sin considerar el costo del terreno, un VNP ( $8 \%$ ) de 355 y $1.101 \mathrm{US} \$ / \mathrm{ha}$, con y sin el costo del suelo, respectivamente, lo que equivale a una renta anual de de 36 y 112 US\$/ha, respectivamente.

Para concretar dichas rentabilidades, por el lado de la silvicultura se requiere realizar podas oportunas y bien ejecutadas y aplicar un régimen de raleos que permita concentrar el potencial de crecimiento del sitio en un número apropiado de árboles de cosecha final de alta calidad, con dos o tres rollizos podados. Por el lado del mercado, debe existir una demanda industrial de este tipo de madera, que esté dispuesta a pagar precios similares a los de $P$. radiata para los rollizos de aptitud aserrable y chapas.

Para una misma condición de productividad de sitio y desde el punto de vista de la economia del productor forestal independiente, la opción de alto valor con $E$. nitens no debiera representar una competencia por uso de suelo forestal con plantaciones de $E$. globulus, donde esta especie logre crecimientos medios por sobre los $20 \mathrm{~m}^{3} / \mathrm{ha}$ año, salvo que las expectativas de precios de los rollizos podados y/o aserrables de $E$. nitens sean muy superiores a los precios empleados en este trabajo.

Bajo una misma condición de productividad de sitio y considerando los supuestos empleados en este trabajo, si el precio de mercado de la madera pulpable de $E$. nitens fuera de $\$ 25.700$ por MR $\left(28,56\right.$ US $\left.\$ / \mathrm{m}^{3} \mathrm{ssc}\right)$, el productor forestal lograría la misma rentabilidad que con plantaciones pulpables de la especie $E$. globulus y sobre este precio sería más rentable $E$. nitens. 
Bajo una misma condición de productividad de sitio y considerando los supuestos empleados en este trabajo, si el precio de mercado de la madera pulpable de $E$. nitens fuera de $\$ 27.600$ por MR $\left(30,7 \mathrm{US} \$ / \mathrm{m}^{3} \mathrm{ssc}\right)$, el productor forestal lograria la misma rentabilidad que invertir en plantaciones de alto valor con $E$. nitens. Sobre este precio debiera privilegiarse el manejo pulpable.

\section{REFERENCIAS}

Baso, C., 2004. Potencialidad del Eucalyptus globulus como Madera Sólida. Universidad de Vigo, Escuela Universitaria de Ingenieria Técnica Forestal. Boletín de Información Técnica N 228. pp 66-72. http://infomadera.net/images/19222.pdf

Bregar, M., 2005. Agricola y Forestal Natalhue Ltda., comunicación personal.

Cabrera, J., 2003. Mercado de E. nitens. Presentación Seminario Forestal "Situación Actual y Proyecciones de Eucalyptus nitens para el Sur de Chile. Valdivia, Agosto, 2003.

http://www.infor.cl/webinfor/pw-nitens/b-nitens/index.htm

Candy; S.; Gerrand, A., 1997. Comparison of Financial Returns from Sawlog Regimes for Eucalyptus nitens Plantations in Tasmania. Tasforests, 9:(35-50).

Corporación Nacional Forestal CONAF, 2004. Tabla de Costos 2005, www.conaf.cl

Cubbage, F.; Mac Donagh, P; Noemi, M.; Siry, J.; Sawinski, J.; Ferreira, A.; Hoeflich, V.; Ferreira, G.; Morales, V.; Rubilar, R.; Alvarez, J.; Donoso, P., 2005. Comparative Timber Investment Returns for Selected Plantations and Native Forests in South America and the Southern United States. Abstract for 2005 SOFEW Conference, Baton Rouge, Louisiana, USA; April 18-20, 2005

Donnelly, R.; Flynn, R.; Shield, E., 2003. The Global Eucalyptus Wood Products Industry. A Progress Report on Achieving Higher Value Utilization. Brochure. http://www.wri-Itd.com/PDFs/ Euc\%20brochure\%202003.pdf

Dunn, F., 2003. Prescripciones Técnicas Poda y Raleos Comerciales en Eucalyptus nitens. Subgerencia de Desarrollo - Forestal Mininco S.A. Trawü 2003 Mejorando el Negocio Forestal: "Plantando bien el Pino y el Eucalipto". www.plantex.cl/plantex/presentaciones/fernandodunn.pdf

Flynn, R., 2005. Eucalyptus: Having an Impact on the Global Solidwood Industry. Wood Resources International.

http://www.wri-ltd.com/PDFs/Eucalyptus.pdf

Gaunt, J.; Penellum, B.; Mckenzie, M., 2003. Eucalyptus nitens Laminated Veneer Lumber (LVL) Structural Properties. New Zealand Journal of Forestry Science 33(1): 114-125 (2002).

Géldres, E.; Schlatter, J., 2004. Crecimiento de las plantaciones de Eucalyptus globulus Sobre Suelos Rojo Arcillosos de la Provincia de Osorno, Décima Región. Nota Técnica. Bosque 25(1): 95-101, 2004.

Gerrand, A.; Neilsen, W.; Medhurst, J., 1997. Thinning and Pruning Eucalypt Plantations for Sawiog Production in Tasmania. Tasforests, 9:(15-34).

Herranz, P., 2004. Forestal y Agricola Monteáguila S.A., comunicación personal. 
Hubbard, W.; Abt, R.; Duryea, M.; Jacobson, M., 1998. Estimating the Profitability of Your NonTimber Forestland Enterprise. University of Florida. IFAS Extension. Circular 836. http://edis.ifas.ufl.edu/pdffiles/FR/FR01500.pdf

Instituto Forestal INFOR, 2000. Modelo de Crecimiento para Eucalipto en Chile. Avances de Investigación. Informe Técnico $\mathrm{N}^{\circ} 148$.

Instituto Forestal, 2003a. "Misión Tecnológica Australia de Productores de Eucalyptus nitens de la Región de Los Lagos". Documento de Trabajo Interno. 67 pp. http://www.infor.cl/webinfor/pw-nitens/bnitens/index.htm

Instituto Forestal. 2003b. Boletin de Precios Forestales. Octubre 2003. 14 pp

Instituto Forestal, 2004a. Eucalyptus nitens en Chile: Economia y Mercado. Proyecto FDI CORFO Opciones Productivas: Industria y Mercado. Informe Técnico 166. 55 pp

Instituto Forestal, 2004b. Gira Nacional 2004: Eucalyptus nitens una Opción de Alto Valor para Chile, 11. 12 y 13 de agosto de 2004. Documento de Trabajo Interno. 41 pp.

http://www.infor.cl/webinfor/pw-nitens/b-nitens/index.htm

Instituto Forestal, 2005a. Exportaciones Forestales Chilenas, 2004. Boletin Estadistico 99. 168 pp

Instituto Forestal, 2005b. Exportaciones Forestales Chilenas,

Marzo 2005. Boletin Estadístico. 106 pp

Klemperer, D., 1996. Forest Resource Economics and Finance. McGraw-Hill. 551pp.

Leiva, F., 2004. CEFOR-UACH, comunicación personal.

Ludwig, G., 2004. Agricola y Forestal San Alejandro Ltda., comunicación personal.

Ludwig, G., 2005. Agricola y Forestal San Alejandro Ltda., comunicación personal.

Mckenzie; H.; Turner, J.; Shelbourne, J., 2003. Processing Young Plantation-Grown Eucalyptus nitens for Solid-Wood Products. 1: Individual-Tree Variation in Quality and Recovery of Appearance-Grade Lumber and Veneer. New Zealand Journal of Forestry Science 33(1): $62-78$ (2003)

Menzel, M., 2005. Agricola y Forestal El Trébol Ltda., comunicación personal.

Mininco, 2003. Norma de Productos Aserrables para Pino radiata.

http://www.mininco.cl/icorporativa/NORMA_ASERRABLE_2003_09.doc

Montagu, K.; Kearney, D.; Smith, G., 2003. Pruning Eucalypts. The Biology and Silviculture of Clear Wood Production in Planted Eucalypts RIRDC Publication No 02/152. RIRDC Project No PN.99.2011. www.rirdc.gov.au/reports/AFT/02-152.pdf

Nolan, G.; Greaves, B.; Washusen, R.; Parsons, M.; Jennings, S., 2005. Eucalypt Plantations for Solid Wood Products in Australia - A Review Olf you don't prune it, we can't use it'. Forest \& Wood Products Research \& Development Corporation

www.fwprdc.org.au/content/pdfs/PN04.3002.pdf

Prado, J. A. y Barros, S., 1989. Eucalyptus Principios de Silvicultura y Manejo. INFOR-CORFO. 199 pp. 
Provoste, F., 2004. Descripción Técnica y Económica de un Sistema de Cosecha Tradicional de Eucalyptus sp. con la Incorporación de Descortezado Mecánico en la X Región. Tesis, Universidad Austral de Chile, Facultad de Ciencias Forestales, Escuela de Ingenieria Forestal.

Shield, E., 2002. Utilisation of Plantation-Grown Eucalyptus : New Resources ... New Approaches International Symposium on Eucalyptus Plantations. Guangzhou - Zhaoqing. Guangdong. China. September 2002.

Superintendencia de Administradora de Fondos de Pensiones SAFP, 2005. Boletin Estadistico N 184. http://www.safp.cl/inf_estadistica/index.html

Zapata, A., 2001. Efectos de las Variables de Establecimiento Inicial en el Crecimiento y la Rentabilidad en Plantaciones de E.globulus y E. nitens. In: Actas Simposio Internacional IUFRO. Desarrollando el Eucalipto del Futuro. 
